\title{
Outcome of surgically treated acetabular fractures: risk factors for postoperative complications and for early conversion to total hip arthroplasty
}

Zanna Luigi ( $\square$ luigizanna90@gmail.com )

University of Florence

Ceri Lorenzo

University of Florence

Scalici Gianluca

University of Florence

Boncinelli Debora

University of Florence

Buzzi roberto

University of Florence

Pietro De Biase

University of Florence

\section{Research Article}

Keywords: Acetabular fracture, ORIF, Hip arthroplasty, Polytrauma, Postoperative complications

Posted Date: December 21st, 2021

DOI: https://doi.org/10.21203/rs.3.rs-1183247/v1

License: (9) (i) This work is licensed under a Creative Commons Attribution 4.0 International License.

Read Full License 


\section{Abstract}

Introduction: Acetabular fractures (AFxs) are rare injuries considering their incidence. The gold standard of treatment is open reduction and internal fixation (ORIF). Surgical treatment represents a challenge for orthopedic surgeons. Our purpose is to assess the short- to medium-term functional outcomes and complications of surgically treated AFx. We analysed the factors influencing clinical outcomes, the incidence of complications and the predictors of conversion in total hip arthroplasty (THA).

Materials and Methods: We retrospectively analysed 102 patients with AFx treated between December 2017 and September 2020. The inclusion criteria were AFx treated with ORIF and more than 12 months of follow-up (FU). We evaluated the quality of reduction with X-ray measuring residual displacement, classified into 3 groups (Matta Radiological Score). Postoperative superficial and deep infections, neurological sequelae and deep vein thrombosis were documented. X-rays were performed to confirm the adequacy of fixation and complications. At the final FU, the radiographs were graded according to Matta's Radiological Outcome Grading, and the clinical outcomes were graded using the Oxford Hip Score (OHS).

Results: Of 102 patients, 62 patients were enrolled. The statistical analysis revealed that OHS was influenced by quality of reduction ( $P=0.033)$, injury severity score (ISS) $(P=0.005)$ and age ( $>75$ years) $(P=0.029)$. A significant correlation between the patient's $B M I$ and the OHS was recorded. The late sequelae were heterotopic ossification (HO) in 13 patients, osteoarthritis (OA) in 22 and avascular necrosis $(\mathrm{AVN})$ in 4 . HO was significantly affected by the posterior approach $(P=0.031)$ and by an ISS > $15(P=0.0003)$. The analysis showed a correlation between AVN and posterior hip dislocation $(P=0.004)$. $O A$ had a correlation with postoperative quality of reduction $(P=0.014)$. Eight patients required THA. Comparing patients with and without THA, a significant correlation between THA and posterior dislocation $(P=0.022)$, isolated posterior wall fracture $(P=0.039)$ and ISS $>15(P=0.029)$ was recorded.

Conclusion: Despite the improvement of surgical techniques and perioperative care, a high rate of patients with AFx still develop complications and require THA. Identifying negative predictors to help the management of fractures in elderly individuals is needed. Furthermore, the presence of negative predictors could represent an indication for primary THA.

\section{Introduction}

Acetabular fractures (AFx) are relatively rare injuries (3/100.000 per year) [1], with relevant growth in developing countries. They usually present a bimodal distribution in younger patients after high-energy traumas and in older patients after a low-energy fall [2]. Surgical treatment with open reduction and internal fixation (ORIF) is considered the gold standard. However, operative treatment of AFx represents a major challenge for orthopedic surgeons. Complications following ORIF are common, including posttraumatic osteoarthritis (OA), chondrolysis, avascular osteonecrosis of the femoral head (AVNFH), heterotopic ossification ( $\mathrm{HO})$ and iatrogenic nerve palsies [3]. When posttraumatic joint disease develops, total hip arthroplasty (THA) may be the only option available [4]. Our hypothesis was that the outcomes 
of surgically treated acetabular fractures are dependent on many factors, and the identification of predictors of poor results and complications in each patient was important to help surgeons in decisionmaking and the management of AFx, especially in geriatric patients. The objective of the study was to assess the short- to medium-term functional outcomes and complications of surgically treated AFx and to analyse the factors that influence clinical outcomes, the incidence of complications and the necessity to perform further surgery to implant a THA.

\section{Material And Methods}

This study is a retrospective analysis of 102 patients treated for closed pelvic trauma with acetabular fracture by a single highly experienced surgeon (PDB) between December 2017 and September 2020 in our trauma center. The inclusion criteria were isolated acetabular fracture surgically treated with ORIF and postoperative follow-up (FU) at least 12 months. Patients with associated pelvic ring injury or open fracture, loss to FU, or an FU shorter than 12 months and those who underwent primary THA were excluded $(x=40)$. All patients were admitted to our emergency department (ED), and a detailed clinical examination and radiological assessment were collected. In patients with posterior hip dislocation (PHD), closed reduction was carried out within 6 hours. The radiological evaluation included plain anteroposterior, oblique Judet radiographs of the pelvis and computed tomography (CT) scans with 3D reconstruction. Demographic data for each patient were collected. The fractures were classified according to both $\mathrm{AO}$ and Judet-Letournel classification. Patients received standard perioperative antibiotic prophylaxis. Surgery was performed under general anaesthesia. We utilized a modified Stoppa approach with a lateral window, ilioinguinal approach, and Kocher-Langenbeck approach with and without trochanter osteotomy according to the fracture pattern. Intraoperative fluoroscopic control was performed to evaluate the quality of reduction and the presence of intraarticular bodies and to avoid metal work that did not violate the hip joint. The Matta Radiological Score was applied to postoperative radiographs: anatomical (0-1 mm of gap), congruent (2-3 mm) or incongruent (>3 $\mathrm{mm})$. The suction drain was maintained for 24-72 hours postoperatively.

Antithromboembolic prophylaxis was maintained until the patient recovered normal gait function. No prophylaxis for $\mathrm{HO}$ was administered. Postoperative rehabilitation was tailored to the patient and the fracture; in the majority of the cases, the patients started rehabilitation consisting of intermittent, painfree quadriceps, hip and knee flexion exercises as early as possible. Toe touch weight bearing was allowed depending on the presence of other fractures. Partial weight bearing was permitted at 6 weeks, progressing to weight bearing as tolerated at 8-12 weeks. The clinical and radiological FU was performed at one, two, three and six months and then once a year. $\mathrm{HO}$ was classified according to Brooker et al [5], and AVNFH was classified according to Ficat and Arlet [6]. Postoperative superficial and deep infections, neurological sequelae, deep vein thrombosis, pulmonary embolism and anaemia were documented. FU radiographs at one year or more were graded according to Matta's Radiological Outcome Grading [7], and functional outcome was evaluated using the Oxford Hip Score (OHS) [8]. 
Statistical analysis was performed using SPSS statistics software version 25.0 for MACINTOSH (IBM, Armonk, NY). The normal distribution was tested with Kolmogorov-Smirnov's test. Descriptive statistics (mean, standard deviation etc.) were used to describe the patients' variables and clinical, functional and radiological outcomes. Categorical variables were assessed using the chi-square test or Fisher's exact test for statistical significance. The Mann-Whitney test was used to evaluate the impact of patient weight body mass index on the final outcome score (OHS). A nonparametric test used to measure the strength of association between the two variables, quality of fracture reduction (Matta Radiological Score) and Matta's Radiological Outcome Grading, was Spearman's rho coefficient. P-values $<0.05$ were considered statistically significant.

\section{Results}

According to the inclusion criteria, 62 patients were enrolled: 47 males and 15 females. The mean age of the group was 53,4 years with a normal distribution $(P=0,95)$. The right side was affected in 27 patients, and the left side was affected in 35 patients. Patients characteristics are summarized in table 1. Road traffic accidents (RTAs) were the principal trauma cause of trauma (Table 2). The mean injury severity score (ISS) was $12,9 \pm 9,6$, and 27 patients (43,5\%) sustained polytrauma. The trauma-related injuries and fracture types are reported in Tables 3 and 4, respectively. In line with the fracture pattern, the modified Stoppa approach with iliac window, Kocher-Langenbeck and Ilioinguinal were used in 32, 26 and 4 patients, respectively. The postoperative quality of reduction reported according to the Matta radiological score was anatomical, congruent and incongruent for 45,14 and 3 hips, respectively.

\section{Failures and Complications}

Complications related to trauma and surgical treatment are shown in Table 5. The 7 sciatic deficits, all present before the surgery, were in patients with posterior wall, posterior column + posterior wall and transverse + posterior wall fractures. Fifteen patients received blood transfusions. We reported 1 case of sciatic paralysis with no recovery and two with partial restoration of external popliteal sciatic function. The patient with deep infection was treated by debridement and partial removal of hardware with prolonged antibiotic therapy. We reported a case of synthesis failure requiring a second surgery based on an ileo-inguinal approach. After the final radiographic assessment, postoperative complications were $\mathrm{HO}$ in 13 patients, posttraumatic OA in 22 and AVNFH in 4. 
The OHS was excellent in 30 hips, good in 15, fair in 4 and poor in 13. The mean score was 35,39 points (range 7-48), with a normal distribution $(P=0.067)$. The OHS was significantly influenced by the quality of reduction (Fig. 1,2), ISS and age of the patient (>75 years). The sex, type of fracture and presence of PHD at ED admission did not significantly correlate with clinical outcome (Table 6). Statistical analysis reported a significant association between the patient's BMI and the OHS $(P<0.0001)$. A high BMI was correlated to a worse outcome. At the last FU, the outcome, according to Matta's Radiological Outcome Grading, was excellent, good, fair and poor in 20, 23, 11, and 8 hips, respectively. A significant correlation between the quality of postoperative reduction and Matta's Radiological Outcome Grading at final radiographs was revealed using the Spearman Rho coefficient $(0.411$ with $P<0.0001)$. Among the postoperative complications, $\mathrm{HO}$ was significantly affected by the Kocher-Langenbeck surgical approach $(P=0.031)$ and by an ISS value $>15(P=0.0003)$. Fracture pattern and posterior dislocation were not significant ( $P=0.88$ and $P=0.59$, respectively). All 4 patients with AVNFH suffered from PHD at ED admission. The statistical analysis reported a significant correlation between AVNFH and PHD $(P=0.004)$. Regarding $\mathrm{OA}$, we reported a significant correlation with the postoperative quality of $\mathrm{AFx}$ reduction $(P=0.014)$. The patients with Matta Radiological Score rated as congruent and incongruent developed OA more frequently than patients with anatomical reduction.

\section{Total Hip Arthroplasty}

Eight patients with poor radiological and clinical outcomes required THA that was performed using the MAKO-assisted surgical technique. The mean OHS was 13 (poor), and the mean Matta's Radiological Outcome grading was 2.5. Four of these patients presented AVNFH, 3 presented severe HO (Brooker grade III/IV) and four presented advanced OA. Comparing patients who underwent THA with patients who did not require this procedure, we reported a significant correlation between THA and PHD $(P=0.022)$, isolated posterior wall fracture $(P=0.039)$ (Fig. 3$)$ and ISS score value greater than 15 points $(P=0.029)$. The quality of reduction was not associated with secondary THA $(P=0.68)$. In patients who had undergone THA, we observed a relevant increase in the mean OHS (38.2) and recovery from basic and advanced activities of daily living.

\section{Discussion}

Pelvic and AFx are one of the most challenging surgeries for orthopedic trauma surgeons. The complex pelvic and acetabular anatomy, difficulty of surgical approach, proximity to the hip joint and frequent involvement of surrounding soft tissues and neurovascular structures represent crucial factors. The mean age of our patients (53,4 years) was slightly higher and had a normal distribution differently according to the bimodal distribution reported in the literature [9-10]. High velocity trauma and falling from height represent the major cases of injury in our cohort $(61,3 \%)$. Our findings are slightly different from other studies in which RTA is the major traumatic mechanism [9-11]. The mean ISS of our cohort was 12,9 , slightly lower than that of other cohorts in the literature $[1,11,14]$, but the rate of patients 
with an ISS higher than 15 was remarkable (26,2\%). Moreover, patients with higher ISS scores were excluded for associated pelvic ring fractures. Posterior wall and both column fractures were the most frequent in our patients $(67,7 \%)$. These data are in close agreement with the results reported by several authors $[1,10,11,12]$. Overall, $27.4 \%$ of our patients were evaluated as fair and poor according to $\mathrm{OHS}$. This is comparable to data from Giannoudis et al meta-analysis [1]: 16 studies (1610 patients) used the modified Merle d'Aubigné score (MDAs) to assess the functional results, and five (600 patients) recorded the Harris hip score (HHS). Using the MDAs, $20,6 \%$ of the population was scored as fair and poor. With HHS, $26,8 \%$ of patients had fair and poor results [1]. According to HHS, Mears et al [14] and Matta [7] reported $28 \%$ and $24 \%$ fair and poor outcomes, respectively. The gold standard for the treatment of AFx is surgery because of damage to the cartilaginous surface of the bone. The most relevant goal of the surgeon is to restore a smooth and gliding hip surface with stable fixation. Several aspects affecting the outcome of AFx contribute to poor results regardless of the treatment method. The main known predictors are type of fracture, quality of reduction, osteochondral defects in the acetabulum or the femur, and the most common complications are $\mathrm{OA}, \mathrm{AVNFH}, \mathrm{HO}$, nervous injuries and infection. The accuracy of reduction correlates strongly with outcome [14], and return to preinjury functional activity is not common. In the present study, 45 out of 62 hips had excellent or good medium-term OHS, which is similar to the results reported in other publications $[11,14,15]$. The quality of the reduction represents a crucial factor; excellent or good functional outcomes can be obtained in the majority of patients [16] if anatomical reduction is achieved [17]. In our study, 45 out of 62 hips had anatomical reduction according to Matta Radiological criteria. According to the literature [17], anatomical reduction has a significant influence on $\mathrm{OHS}$ in our population $(P=0.033)$, determining good and excellent functional results. Eighty percent of our patients with an anatomical Matta radiological score had good and excellent outcomes. The OHS was significantly influenced by ISS, patient age ( $>75$ years) and BMI. Patients with an ISS $<15$ exhibit a better functional outcome at the final FU. Many authors have reported no significant differences in the mean ISS between patients with good and poor results [12,18]. Some studies [19] did not analyse ISS but underlined the negative effects of associated musculoskeletal injuries on the clinical score. The negative impact of the patient's BMI on OHS in our population was confirmed by the literature. Obesity affects postoperative complications and outcomes of surgically treated AFx. Madhav et al [20] retrospectively identified and stratified 169 patients according to their BMI. The obese subjects $(\mathrm{BMI}>30)$ were 2.1 times more likely to have blood loss $>750 \mathrm{~mL}$ and 2.6 times more likely to have DVT. The patients with $\mathrm{BMI}>40$ were five times more prone to have a wound infection. We divided patients into two groups according to their age ( $>75$ and $<75$ years) [21]. The older group developed worse clinical outcomes. The negative influence of age on clinical results is confirmed in the literature [22]. Risk factors such as sex, type of fracture and presence of PHD at ED admission did not have any significant impact on the postoperative clinical score [11]. The quality of AFx postoperative reduction is the most important predictor not only of clinical function but also of radiological grade and development of $O A$. We report that a postoperative displacement greater than 2 $\mathrm{mm}$ has a significant effect on both the functional score and Matta's Radiological Outcome Grading at the final FU. The complications of AFx documented in the literature include infection, nerve injury, $\mathrm{HO}$, thromboembolic issues, OA, AVNFH, malunion, and nonunion $[1,27]$. The incidence of infection 
was approximately 2-5\% [23-25]. Giannoudis et al [1] reported a 4.4\% surgical site infection. The risk of infection was higher in patients requiring ICU care, obese patients and patients with associated MorelLavallee lesions. We report one case of deep infection of the surgical site associated with fistula. We recorded 7 cases $(11,5 \%)$ of DVT and no cases of pulmonary embolism (PE). In the literature, the perioperative incidence of DVT is debated. Wang et al [26] investigated the incidence and risk factors for DVP and described an incidence of $29.09 \%$ in patients with AFx. Many studies have reported that AFX is associated with a broad spectrum of incidences of DVT, between $2 \%$ and $33,7 \%$, and PE, between $0.99 \%$ and $21.4 \%$ [27-28]. We collected seven cases of sciatic nerve palsy at ED admission (11\% of our population). No cases of iatrogenic nerve palsy were recorded. Similarly, Briffa et al [17] and Meena et al [11] reported $12 \%$ sciatic nerve palsy with three iatrogenic injuries. In contrast, Lehmann et al [29] reported $4 \%$ nerve injuries at hospital admission and $7 \%$ at discharge. The literature indicates that the incidence of patients with neurological deficits, including iatrogenic injuries after acetabulum fracture, is $5 \%$ to $33 \%[14,32,33]$. Patients with posterior acetabular wall fracture and hip dislocation most commonly develop traumatic nerve injuries [11]. In our cohort, we collected $60 \%$ late complications. Despite a prompt closed reduction (within 6 hours), $6 \%$ of our patients suffered from $A V N F H$, in line with the incidence reported in the literature (3-11\%) $[1,17,34]$. In our analysis, AVNFH development was significantly related to the presence of PHD $(P=0.004)$. To our knowledge, many authors have described AVNFH as a complication after traumatic PHD and posterior acetabular fracture-dislocations [35-36], highlighting the importance of hip reduction timing with an increased risk 12 hours after the injury [35-36]. HO represents a common problem in AFx, with many controversies regarding its aetiology. The incidence of $\mathrm{HO}$ in our population is $20,1 \%$. We have not administered indomethacin as a protective drug because its efficacy is still controversial [39-40]. The rate of this complication is in accordance with the literature $[1,39]$. The analysis of our cohort reports posterior surgical approach $(P=0.031)$ and ISS value $>15(P=0.0003)$ as significant risk factors. In the literature, there are still conflicting results in terms of risk factors. Some authors reported posterior approach $[1,15]$, male sex, ICU admission, ISS and associated head or thoraco-abdominal traumatic injury [15,41], delayed surgery and multiple fractures [42]. Other studies did not correlate these factors with the formation of $\mathrm{HO}$ [43-44]. The most common complication, emphasized by Matta et al. [3], was posttraumatic $O A$. The incidence in the literature ranges between $20 \%$ and $50 \%[1,11,17,45]$. The overall incidence of $\mathrm{OA}$ in our series is $34,5 \%$. Analysis of predictive risk factors for $\mathrm{OA}$ revealed a significant correlation with postoperative quality of reduction of the AFx $(P=0.014)$. Giannoudis et al [1] reported that the incidence of $O A$ is related to the quality of reduction. Among the seven studies investigated by Ziran et al., when the reduction was anatomical $(<2 \mathrm{~mm})$, the incidence was $13.2 \%$. However, if the reduction was not satisfactory (>2 mm), the incidence of OA increased to $43.5 \%$ [23]. Many other authors have highlighted the importance of anatomical reduction in the development of OA [46-47]. OA and AVNFH are both predictors for conversion to THA. In our study, eight patients (12,9\%) required THA performed by the robot-assisted MAKO technique. Four of them had developed AVNFH, 3 HO (Brooker IV) and 4 OA. Several studies showed similar rates of THA, between $18 \%$ and $24 \%$, despite different FU intervals $[18,39]$. In our population, anatomical reduction was obtained in more than $70 \%$ of fractures, which probably explains the relatively low rate of conversion to THA. Our analysis revealed a 
significant correlation between THA and PHD $(P=0.022)$, isolated posterior wall fracture $(P=0.039)$ and ISS score $>15$ points $(P=0.029)$. In the literature, the main negative predictors included patient age, not anatomical reduction, incongruence of the acetabular roof [16], femoral head cartilage lesion, acetabular cartilage impaction, delayed reconstruction, and relocation of a PHD later than 12 hours [48-49]. Tannast et al [47] identified additional risk factors, including hip fracture dislocation, free intra-articular fragments, and involvement of the posterior acetabular wall. In this regard, we suppose that PHD and posterior AFx indicate high-intensity trauma with transmission of high-intensity direct forces occurring in acetabular wall fractures. This traumatic mechanism can determine comminution and severe impaction of the posterior wall, cartilage damage and vascular femoral head supply interruption. These factors associated with soft tissue lesions contribute to poor clinical outcome and predict conversion to THA. The main limits of this study are its retrospective nature associated with a relatively small sample of patients and medium-term FU. While the numbers are not ideal, they represent an important value if the short timeline (2 years) is taken into consideration. Furthermore, positive points of this analysis are the single highly experienced surgeon series from a single hospital associated with homogeneous rehabilitation, which allows us to directly compare and validate results and the application of international score (OHS) collected at the last FU, which permits a direct comparison with different reports in the literature. Despite the improvement of surgical techniques and perioperative care, a high rate of patients with AFx still develop complications and need THR. The identification of predictors of poor clinical outcome and complications in each patient is important to help surgeons in the decision-making and management of AFx, especially in geriatric patients. In our experience, the presence of predictors of failure in this population could represent an indication for primary THA.

\section{Conclusions}

Data from the case series study confirm that a good surgical reduction of AFx can provide a good to excellent result over a medium period of FU. However, in obese or elderly patients, this may not be sufficient to obtain a satisfactory result. Association between age $>75$ years, obesity and fracture of posterior column or wall or PHD may suggest addressing the patient with a primary prosthesis or with a reduction and synthesis plus a primary prosthetic approach to avoid complications and later surgery in a short interval. AVNFH is still not easily predicted, so authors do not suggest primary prostheses in younger patients with PHD associated with posterior column or posterior wall fracture.

\section{Declarations}

Ethical approval and consent to participate: This study was approved by our institutional review board. Informed consent was obtained from all patients for inclusion in the study.

Consent for publication: Informed consent was obtained from all patients for inclusion in the study.

Availability of data and materials: The datasets generated and/or analyzed during the current study are not publicly available but are available from the corresponding author upon reasonable request because 
there are private data of the patients.

Competing interests: Not applicable.

Funding: Not applicable.

Author contributions: LZ, LC and PDB contributed to the study conception and design. Material preparation, data collection and analysis were performed by $L Z, S G, L C$ and DB. The first draft of the manuscript was written by LZ and LC, and all authors commented on previous versions of the manuscript. All authors read and approved the final manuscript.

Acknowledgements: Not applicable.

\section{Abbreviations}

Acetabular fractures (AFx)

Open reduction and internal fixation (ORIF)

Total hip arthroplasty (THA)

Follow-up (FU)

Oxford Hip Score (OHS)

Injury Severity Score (ISS)

Avascular necrosis (AVN)

Osteoarthritis (OA)

Heterotopic Ossification (HO)

Avascular osteonecrosis of the femoral head (AVNFH)

Emergency Department (ED)

Posterior hip dislocation (PHD)

Computed Tomography (CT)

Body Mass Index (BMI)

Merle d'Aubigné score (MDAs)

Harris hip score (HHS) 


\section{References}

1. Giannoudis PV, Grotz MRW, Papakostidis C, Dinopoulos H. Operative treatment of displaced fractures of the acetabulum. A meta-analysis. J Bone Joint Surg Br. 2005;87:2-9.

2. Henry PDG, Kreder HJ, Jenkinson RJ. The Osteoporotic Acetabular Fracture. Orthopedic Clinics of North America. 2013;44:201-15.

3. Matta JM, Mehne DK, Roffi R. Fractures of the acetabulum. Early results of a prospective study. Clin Orthop Relat Res. 1986;:241-50.

4. Wu ES, Jauregui JJ, Banerjee S, Cherian JJ, Mont MA. Outcomes of delayed total hip arthroplasty in patients with a previous ipsilateral acetabular fracture. Expert Rev Med Devices. 2015;12:297-306.

5. Brooker AF, Bowerman JW, Robinson RA, Riley LH. Ectopic ossification following total hip replacement. Incidence and a method of classification. J Bone Joint Surg Am. 1973;55:1629-32.

6. Ficat RP. Idiopathic bone necrosis of the femoral head. Early diagnosis and treatment. J Bone Joint Surg Br. 1985;67:3-9.

7. Matta JM. Fractures of the acetabulum: accuracy of reduction and clinical results in patients managed operatively within three weeks after the injury. J Bone Joint Surg Am. 1996;78:1632-45.

8. Wylde V, Learmonth ID, Cavendish VJ. The Oxford hip score: the patient's perspective. Health Qual Life Outcomes. 2005;3:66.

9. Munshi N, Abbas A, Gulamhussein MA, Mehboob G, Qureshi RA. Functional outcome of the surgical management of acute acetabular fractures. Journal of Acute Disease. 2015;4:327-30.

10. Petsatodis G, Antonarakos P, Chalidis B, Papadopoulos P, Christoforidis J, Pournaras J. Surgically treated acetabular fractures via a single posterior approach with a follow-up of 2-10 years. Injury. 2007;38:334-43.

11. Meena UK, Tripathy SK, Sen RK, Aggarwal S, Behera P. Predictors of postoperative outcome for acetabular fractures. Orthopaedics \& Traumatology: Surgery \& Research. 2013;99:929-35.

12. Deo SD, Tavares SP, Pandey RK, El-Saied G, Willett KM, Worlock PH. Operative management of acetabular fractures in Oxford. Injury. 2001;32:581-6.

13. Dunet B, Tournier C, Billaud A, Lavoinne N, Fabre T, Durandeau A. Acetabular fracture: Long-term follow-up and factors associated with secondary implantation of total hip arthroplasty. Orthopaedics \& Traumatology: Surgery \& Research. 2013;99:281-90.

14. Mears DC, Velyvis JH, Chang C-P. Displaced acetabular fractures managed operatively: indicators of outcome. Clin Orthop Relat Res. 2003;:173-86. 
15. Chiu F-Y, Chen C-M, Lo W-H. Surgical treatment of displaced acetabular fractures -72 cases followed for $10(6-14)$ years. Injury. 2000;31:181-5.

16. Letournel E. Acetabulum fractures: classification and management. Clin Orthop Relat Res. 1980;:81106.

17. Briffa N, Pearce R, Hill AM, Bircher M. Outcomes of acetabular fracture fixation with ten years' followup. THE JOURNAL OF BONE AND JOINT SURGERY. 2011;93:8.

18. Clarke-Jenssen J, Røise O, Storeggen SAØ, Madsen JE. Long-term survival and risk factors for failure of the native hip joint after operatively treated displaced acetabular fractures. The Bone \& Joint Journal. 2017;99-B:834-40.

19. Moed BR, Yu PH, Gruson KI. FUNCTIONAL OUTCOMES OF ACETABULAR FRACTURES: The Journal of Bone and Joint Surgery-American Volume. 2003;85:1879-83.

20. Karunakar MA, Shah SN, Jerabek S. Body mass index as a predictor of complications after operative treatment of acetabular fractures. J Bone Joint Surg Am. 2005;87:1498-502.

21. Ouchi Y, Rakugi H, Arai H, Akishita M, Ito H, Toba K, et al. Redefining the elderly as aged 75 years and older: Proposal from the Joint Committee of Japan Gerontological Society and the Japan Geriatrics Society: Redefining elderly: Proposal from Japan. Geriatr Gerontol Int. 2017;17:1045-7.

22. Wollmerstädt J, Pieroh P, Schneider I, Zeidler S, Höch A, Josten C, et al. Mortality, complications and long-term functional outcome in elderly patients with fragility fractures of the acetabulum. BMC Geriatr. 2020;20:66.

23. Ziran N, Soles GLS, Matta JM. Outcomes after surgical treatment of acetabular fractures: a review. Patient Saf Surg. 2019;13:16.

24. Suzuki T, Morgan SJ, Smith WR, Stahel PF, Gillani SA, Hak DJ. Postoperative surgical site infection following acetabular fracture fixation. Injury. 2010;41:396-9.

25. Li Q, Liu P, Wang G, Yang Y, Dong J, Wang Y, et al. Risk Factors of Surgical Site Infection after Acetabular Fracture Surgery. Surg Infect (Larchmt). 2015;16:577-82.

26. Wang P, Kandemir U, Zhang B, Wang B, Li J, Zhuang Y, et al. Incidence and Risk Factors of Deep Vein Thrombosis in Patients With Pelvic and Acetabular Fractures. Clin Appl Thromb Hemost. 2019;25:107602961984506.

27. Fishmann AJ, Greeno RA, Brooks LR, Matta JM. Prevention of deep vein thrombosis and pulmonary embolism in acetabular and pelvic fracture surgery. Clin Orthop Relat Res. 1994;:133-7. 
28. Kim J-W, Oh C-W, Baek S-G, Lee B-J, Hong H-P, Min W-K, et al. The incidence and the risk factors of venous thromboembolism in Korean patients with pelvic or acetabular fractures. Journal of Orthopaedic Science. 2014;19:471-7.

29. Lehmann W, Hoffmann M, Fensky F, Nüchtern J, Großterlinden L, Aghayev E, et al. What Is the Frequency of Nerve Injuries Associated With Acetabular Fractures? Clin Orthop Relat Res. 2014;472:3395-403.

30. Negrin LL, Seligson D. Results of 167 consecutive cases of acetabular fractures using the KocherLangenbeck approach: a case series. J Orthop Surg Res. 2017;12:66.

31. Moed BR, Carr SEW, Watson JT. RESULTS OF OPERATIVE TREATMENT OF FRACTURES OF THE POSTERIOR WALL OF THE ACETABULUM: The Journal of Bone and Joint Surgery-American Volume. 2002;84:752-8.

32. Milenkovic S, Mitkovic M, Mitkovic M. Avascular necrosis of the femoral head after traumatic posterior hip dislocation with and without acetabular fracture. Eur J Trauma Emerg Surg. 2020. https://doi.org/10.1007/s00068-020-01495-x.

33. Hougaard K, Thomsen PB. Traumatic posterior dislocation of the hip ?Prognostic factors influencing the incidence of avascular necrosis of the femoral head. Arch Orth Traum Surg. 1986;106:32-5.

34. Massoud EIE. Neglected traumatic hip dislocation: Influence of the increased intracapsular pressure. WJO. 2018;9:35-40.

35. Kellam P, Ostrum RF. Systematic Review and Meta-Analysis of Avascular Necrosis and Posttraumatic Arthritis After Traumatic Hip Dislocation: Journal of Orthopaedic Trauma. 2016;30:10-6.

36. Ahmed G, Shiraz S, Riaz M, Ibrahim T. Late versus early reduction in traumatic hip dislocations: a meta-analysis. Eur J Orthop Surg Traumatol. 2017;27:1109-16.

37. Griffin SM, Sims SH, Karunakar MA, Seymour R, Haines N. Heterotopic ossification rates after acetabular fracture surgery are unchanged without indomethacin prophylaxis. Clin Orthop Relat Res. 2013;471:2776-82.

38. Baschera D, Rad H, Collopy D, Zellweger R. Incidence and clinical relevance of heterotopic ossification after internal fixation of acetabular fractures: retrospective cohort and case control study. J Orthop Surg Res. 2015;10:60.

39. Lannes X, Moerenhout K, Duong HP, Borens O, Steinmetz S. Outcomes of combined hip procedure with dual mobility cup versus osteosynthesis for acetabular fractures in elderly patients: a retrospective observational cohort study of fifty one patients. International Orthopaedics (SICOT). 2020;44:2131-8. 
40. Yeo D-H, Oh J-K, Cho J-W, Kim B-S. Management and Outcome of Patients with Acetabular Fractures: Associated Injuries and Prognostic Factors. Journal of Trauma and Injury. 2019;32:32-9.

41. Forsberg JA, Pepek JM, Wagner S, Wilson K, Flint J, Andersen RC, et al. Heterotopic Ossification in High-Energy Wartime Extremity Injuries: Prevalence and Risk Factors: The Journal of Bone and Joint Surgery-American Volume. 2009;91:1084-91.

42. Elhassan Y, Abdelhaq A, Piggott RP, Osman M, McElwain JP, Leonard M. Heterotopic Ossification following acetabular fixation: Incidence and risk factors: 10-year experience of a tertiary centre. Injury. 2016;47:1332-6.

43. d'Heurle A, Archdeacon MT, Hiratzka S, Casstevens C, Finnan R, McCoy B. Do Surrogates of Injury Severity Influence the Occurrence of Heterotopic Ossification in Fractures of the Acetabulum?: Journal of Orthopaedic Trauma. 2016;30:213-6.

44. Firoozabadi R, O'Mara TJ, Swenson A, Agel J, Beck JD, Routt M. Risk Factors for the Development of Heterotopic Ossification After Acetabular Fracture Fixation. Clin Orthop Relat Res. 2014;472:3383-8.

45. Cahueque M, Martínez M, Cobar A, Bregni M. Early reduction of acetabular fractures decreases the risk of post-traumatic hip osteoarthritis? Journal of Clinical Orthopaedics and Trauma. 2017;8:320-6.

46. Øvre S, Madsen JE, Røise O. Acetabular fracture displacement, roof arc angles and 2 years outcome. Injury. 2008;39:922-31.

47. Tannast M, Najibi S, Matta JM. Two to Twenty-Year Survivorship of the Hip in 810 Patients with Operatively Treated Acetabular Fractures: The Journal of Bone and Joint Surgery-American Volume. 2012;94:1559-67.

48. Boudissa M, Ruatti S, Kerschbaumer G, Milaire M, Merloz P, Tonetti J. Part 2: outcome of acetabular fractures and associated prognostic factors-a ten-year retrospective study of one hundred and fifty six operated cases with open reduction and internal fixation. International Orthopaedics (SICOT). 2016;40:2151-6.

49. Kühn K, D'Lima DD, Hashimoto S, Lotz M. Cell death in cartilage. Osteoarthritis and Cartilage. 2004;12:1-16.

\section{Tables}




\begin{tabular}{|ll|}
\hline Variable & Value \\
\hline Age & $53,4+/-14,26(24-81)$ \\
\hline Male & 47 \\
\hline Female & 15 \\
\hline Weight (BMI Kg/m²) & $25,48(18,50-37,50)$ \\
\hline Hip injured & 27 Right, 35 Left \\
\hline Follow-up (Months) & $27,7+/-16,5(12-46)$ \\
\hline Injury severity score (mean) & $12,9+/-9,6(4-57)$ \\
\hline Posterior dislocation (n) & 16 \\
\hline Delay in surgery (Days) & $8,26+/-4,74(1-30)$ \\
\hline Intensive care post-operative (n) & 23 \\
\hline Hospitalization (Days) & $24,58+/-12,36(7-66)$ \\
\hline
\end{tabular}

Table 1. Patients' features

\begin{tabular}{|ll|}
\hline Mechanism & Value \\
\hline Motorcycle accident & 14 \\
Car accident & 14 \\
Fall > 2 mt & 10 \\
Bicycle injury & 6 \\
Pedestrian-struck by vehicle & 6 \\
Other & 12 \\
\hline
\end{tabular}

Table 2. Traumatic mechanism distribution 


\begin{tabular}{|ll|}
\hline Trauma & Value \\
\hline Head & 20 \\
\hline Chest & 23 \\
\hline Intra-abdominal & 14 \\
\hline Upper extremities & 21 \\
\hline Lower extremities & 15 \\
\hline Vertebral & 9 \\
\hline Major vascular & 5 \\
\hline Neurological & 7 \\
\hline
\end{tabular}

Table 3. Associated injury recorded

\begin{tabular}{|ll|}
\hline Type & Value \\
\hline Simple 24 & \\
\hline Posterior Wall & 18 \\
Posterior Column & 0 \\
Anterior Wall & 1 \\
Anterior Column & 5 \\
Transverse & 0 \\
Complex 38 & \\
\hline Posterior column + posterior wall & 1 \\
Transverse + posterior Wall & 5 \\
T-Shaped & 1 \\
Anterior column + hemitransverse posterior & 7 \\
Both columns & 24 \\
\hline
\end{tabular}


Table 4. Fracture pattern distribution according to Judet Letournel

\begin{tabular}{|ll|}
\hline Complication & Value \\
\hline Post-operative Anemia & 15 \\
\hline Deep Vein Thrombosis & 7 \\
\hline Wound Infection & 1 \\
\hline Hardware failure & 1 \\
\hline Nerves lesion & 7 \\
\hline Systemic infection & 3 \\
\hline Heterotopic ossification & 13 \\
\hline Post-Traumatic Arthrosis & 22 \\
\hline AVNF & 4 \\
\hline
\end{tabular}

Table 5. Complications reported in this analysis 


\begin{tabular}{|c|c|c|c|c|}
\hline \multirow[t]{2}{*}{ Factors } & \multicolumn{3}{|l|}{ Oxford score } & \multirow{2}{*}{$\begin{array}{l}P \\
\text { value }\end{array}$} \\
\hline & $\begin{array}{l}\text { Poor + Moderate (0-29) } \\
\text { Total }\end{array}$ & Good + Excellent (30-4 & & \\
\hline \multicolumn{5}{|l|}{ Age } \\
\hline$\geq 75$ & 5 & 3 & 8 & 0.029 \\
\hline$<75$ & 12 & 42 & 54 & \\
\hline \multicolumn{5}{|l|}{ Sex } \\
\hline Male & 13 & 34 & 47 & 1 \\
\hline Female & 4 & 11 & 15 & \\
\hline \multicolumn{5}{|c|}{ Posterior Dislocation } \\
\hline Present & 6 & 10 & 16 & 0.294 \\
\hline Absent & 11 & 35 & 46 & \\
\hline \multicolumn{5}{|c|}{ Quality of reduction } \\
\hline$<2 \mathrm{~mm}$ & 9 & 36 & 45 & 0.033 \\
\hline$>2 \mathrm{~mm}$ & 8 & 9 & 17 & \\
\hline \multicolumn{5}{|c|}{$\begin{array}{l}\text { Injury severity } \\
\text { score }\end{array}$} \\
\hline$\geq 15$ & 9 & 8 & 17 & \\
\hline$<15$ & 8 & 37 & 45 & 0.005 \\
\hline \multicolumn{5}{|c|}{ Type of Fracture } \\
\hline Simple & 7 & 18 & 25 & 0.467 \\
\hline Complex & 9 & 28 & 37 & \\
\hline
\end{tabular}

Table 6. Significant predictors of clinical outcome

Figures 


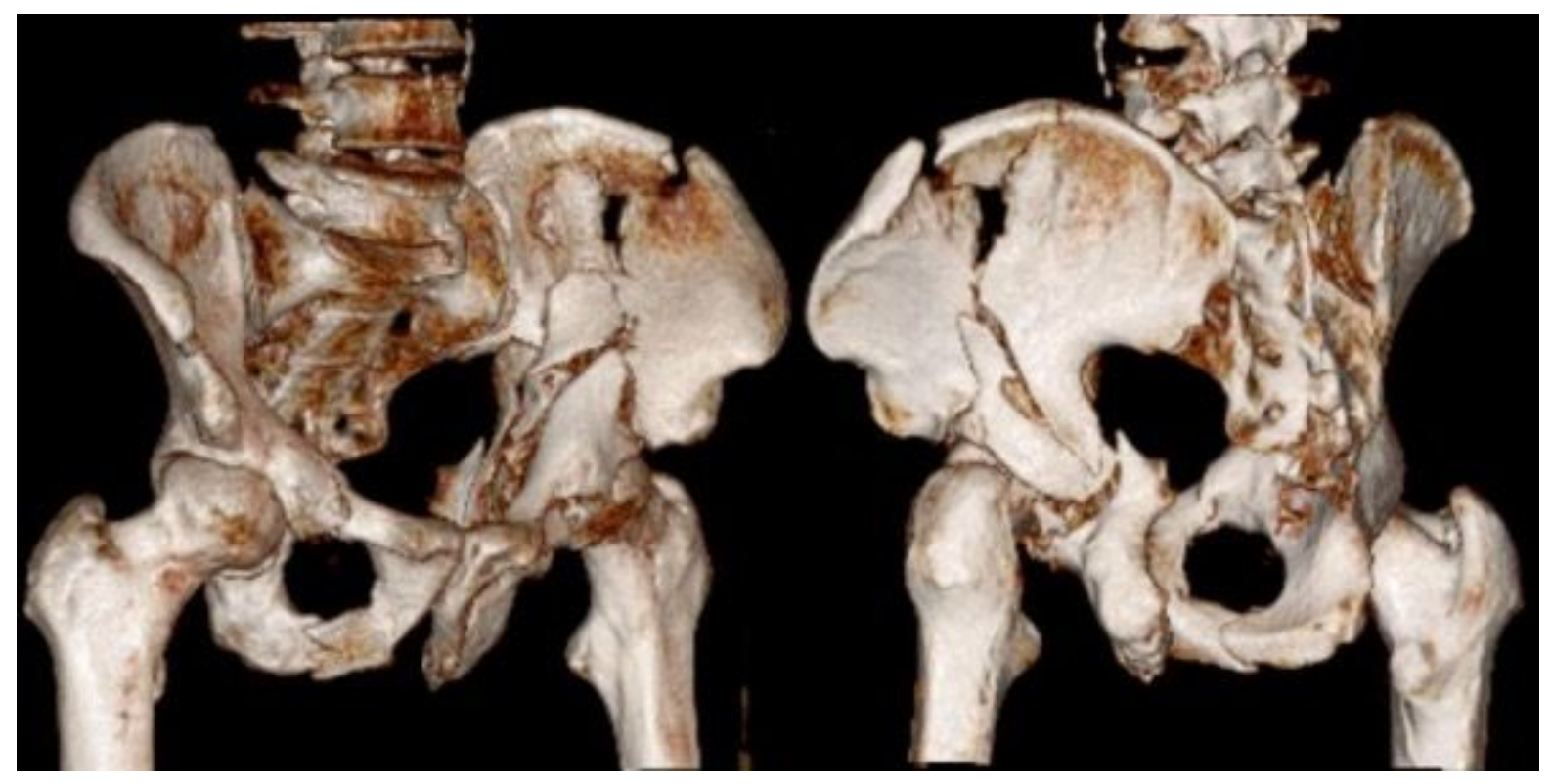

Figure 1

Acetabular both columns fracture

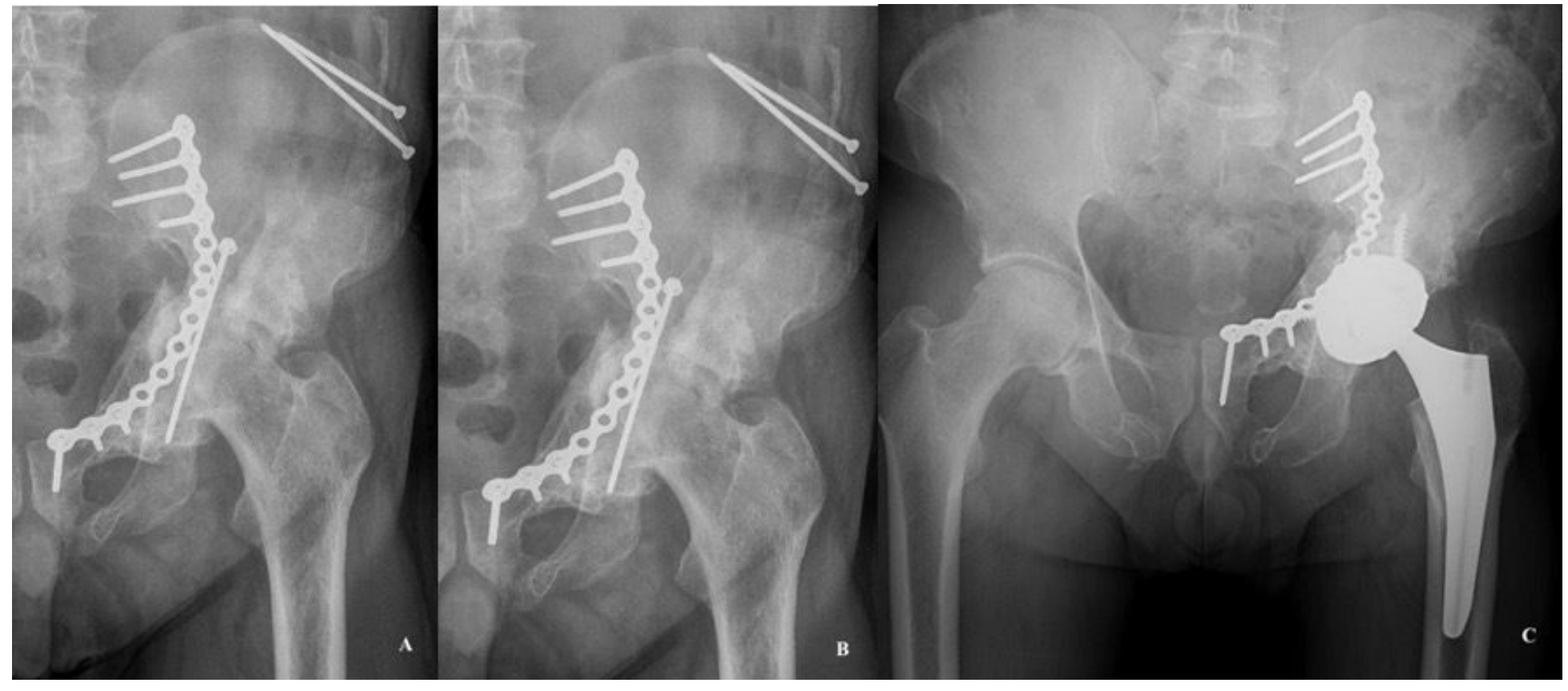

Figure 2

Postoperative incongruent reduction according to Matta Radiological Score (A) Final follow-up X-ray, poor outcome according to Matta's Radiological Outcome Grading and poor clinical OHS (B) Total Hip replacement using MAKO Robot Technique (C) 


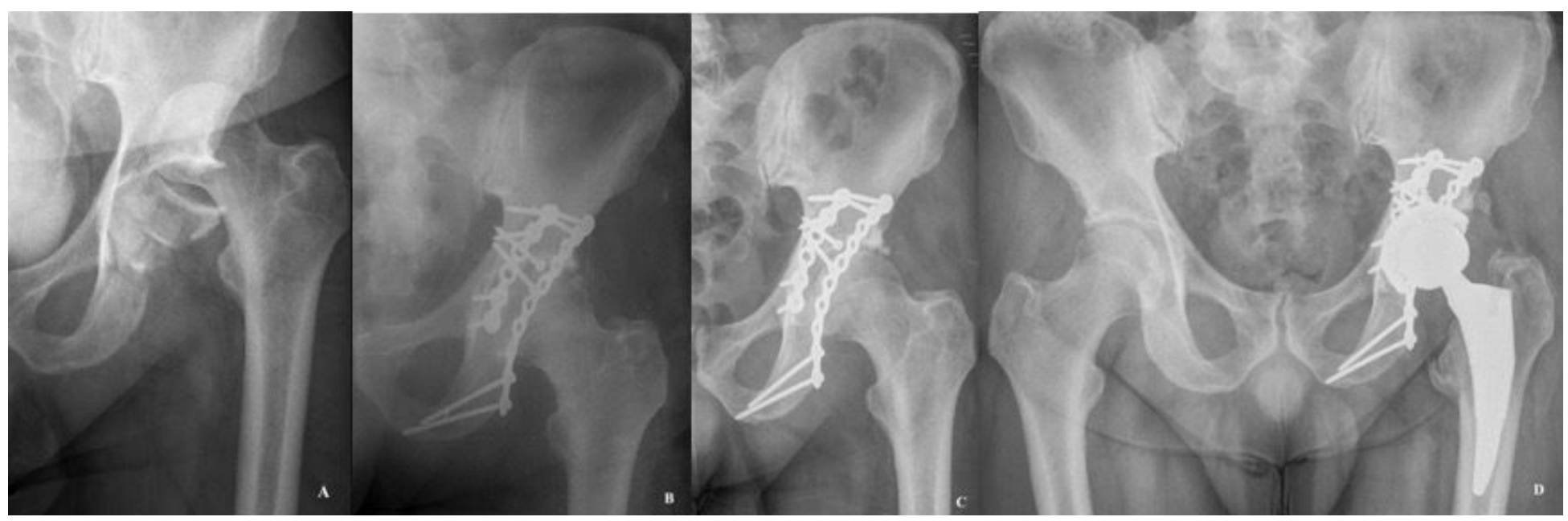

Figure 3

Acetabular posterior wall fracture and posterior dislocation (A) Postoperative anatomical reduction according to the Matta Radiological Score (B) Final follow-up X-ray, poor outcome according to Matta's Radiological Outcome Grading, with femoral head collapse (AVNF) (C) Total hip replacement using the MAKO Robot Technique (D) 